\title{
Re: "Incidence of post-cataract endophthalmitis with intracameral cefuroxime" letter to the editor
}

\author{
Gabriel Katz ${ }^{1} \cdot$ Rita Ehrlich ${ }^{2}$
}

Received: 27 January 2016 / Accepted: 4 February 2016/Published online: 18 February 2016

(C) Springer-Verlag Berlin Heidelberg 2016

Dear Dr. Tan,

Thank you for your interest in our article. We agree that it would be informative to compare the rates of endophthalmitis between patients who did not receive intracameral cefuroxime (ICC) and those that did. As stated in the article, we did not use ICC prior to 2006 and the vast majority of patients received ICC after 2008 since it became our prophylactic treatment policy.

Upon your suggestion, we compared the rates of endophthalmitis between 2000 and 2005 with those between 2009 and 2014 (excluding the transition period between 2006 and 2008). We found an endophthalmitis ratio of 0.077 (18/23, $312)$ in the previous years vs. a ratio of $0.023(5 / 21618)$ in the later. The difference was statistically significant with a $p$ value of 0.02 . The difference is slightly more impressive and significant.

Five of the ten cases of endophthalmitis occurring between 2007 and 2014 did not receive intracameral cefuroxime. One of them occurred in 2007, two cases occurred during 2008, and two cases during 2013.
Three cases of endophthalmitis occurred between 2010 and 2014 in patients that received ICC. As stated above, it is reasonable to assume that the majority of patients received ICC after 2009 at the end of cataract surgery. Of course, this does not fully protect against endophthalmitis. Unfortunately, we cannot report the exact number of patients that did not receive ICC since 2009.

We can only speculate on the difference in the type of organisms of the culture-positive endophthalmitis following the period of the use of ICC. Yet some of the patients that developed endophthalmitis after 2009 did not receive ICC at the end of the cataract surgery due to various reasons that we reported on. Indeed, the difference might be because of better coverage of some of the organisms by the antibiotic injection leading to higher rates of Gram-negative cultures. We feel that the low number of cases prevents us from currently drawing these conclusions.

Dr. Gabriel Katz

Dr. Rita Ehrlich

Gabriel Katz

gabrielkatz.dr@gmail.com

1 Goldschleger Eye Institute, Sheba Medical Center, Ramat Gan 52621, Israel

2 Ophthalmology Department, Rabin Medical Center, Petah Tikva, Israel 